\title{
VISCOELASTIC PROPERTIES OF LOWER EXTREMITY MUSCLES AFTER ELITE TRACK CYCLING SPRINT EVENTS: A CASE REPORT
}

\author{
Sebastian Klich, ${ }^{1, A, B, D}$ Igor Krymski, ${ }^{1,2, A, B}$ Adam Kawczyński', A, B, D \\ 1 University School of Physical Education in Wroclaw, Department of Paralympic Sport, Poland \\ ${ }^{2}$ Polish Cycling Federation, National Team Coach, Andrzeja 1, 05-800 Pruszków, Poland \\ A Study Design; ${ }^{\mathrm{B}}$ Data Collection; ${ }^{\mathrm{D}}$ Manuscript Preparation \\ Address for corpespondence: \\ Sebastian Klich \\ University School of Physical Education in Wrocław, Department of Paralympic Sport \\ Witelona 25a, 51-617 Wrocław, Poland \\ E-mail: sebastian.klich@awf.wroc.pl
}

\begin{abstract}
Ahstract Sprint cycling events require a high level of anaerobic capacity and, therefore, may affect peripheral fatigue throughout exercise-induced muscle damage. In fact, those alterations might decrease power generation. This study was performed on a 23 years old male elite track cyclist taking part in a sprint event. The measurements included power output (W) and cadence $(\mathrm{rpm})$, lactate concentration (La), heart rate (bpm), Rating of Perceived Exertion scale and viscoelastic properties analysis. The present study has shown a new approach to monitor the muscle properties of the lower extremity after $200 \mathrm{~m}$ flying start and repeated sprint races. Therefore, we hypothesized that repeated sprint races might lead to alterations in viscoelastic properties of lower extremity muscles. In track cycling, especially in sprint events, these variations may lead to increased muscle fatigue. Furthermore, training control and monitoring related to the assessment of muscles properties can be a source of counteracting injuries and relieving fatigue.
\end{abstract}

Key Wordls viscoelastic properties, muscle stiffness, creep, $200 \mathrm{~m}$ flying, case report

\section{Introduction}

Sprint performance is dependent on maximal cycling power and is therefore susceptible to muscular fatigue (Martin, Davidson, Pardyjak, 2007). Sprint cycling performance is mainly based on anaerobic metabolism derived from phosphocreatine $(\mathrm{PCr})$ which produces lactate. An increase in lactate accumulation may affect neuromuscular activity and power generation, causing peripheral alterations in muscle fatigue. Moreover, mechanical and morphological properties of lower extremity muscles may influence power production capabilities (Stafilidis, Arampatzis, 2007).

Sprint races are one of the highest performance events in track cycling. They require maximal power output and velocity generation (Klich, Krymski, Michalik, Kawczyński, 2018). S. Dorel et al. (2005) reported that elite $200 \mathrm{~m}$ 
flying start events ranged between $10 \mathrm{~s}$ and $11 \mathrm{~s}$, while in our previous study (Klich et al., 2018), elite track cyclists completed an averaged personal record below $10 \mathrm{~s}$.

Previous studies have reported the negative effect of overloading as muscle degeneration (Kawczyński et al., 2015; Klich et al., 2018). Therefore, an objective research protocol should be used to assess viscoelastic and biomechanical properties in order to prevent the risk of overloading and injuries. Myotonometry is a reliable tool for assessing the viscoelastic properties of muscles (Kawczyński et al., 2018; White, Abbott, Masi, Henderson, Nair, 2018). In previous studies, myotonometry was used to assess muscle stiffness, elasticity and muscle tone (Davidson, Bryant, Bower, Frawley, 2017). Moreover, it provides many quantitative data about muscle belly and tendon stiffness after fatigue (Viir et al., 2007).

To the best of our knowledge, no study to date has used the viscoelastic properties of the lower extremity muscles after a $200 \mathrm{~m}$ flying start and repeated sprints. These observations may provide a crucial understanding of injury prevention, recovery protocols, and physical therapy treatment. Thus, the viscoelastic properties of lower extremity muscles should be investigated after repeated sprint events.

\section{Methods}

\section{Study design}

An observational case study involved six repeated myotonometry measurements, namely: (1) before; (2) after a 200 m flying start; (3-6) following each sprint. Viscoelastic properties of lower extremity muscles (e.g. muscle tone, stiffness, decrement, relaxation time and creep) were investigated in anterior thigh muscles, hamstrings, tibia muscles, and cuff muscles on the right (dominant) side. The participant read and signed an informed consent form approved by the University Research Ethics Committee.

\section{Participant}

An elite track cyclist (age 23 years; body height $176.2 \mathrm{~cm}$; body weight $85.8 \mathrm{~kg} ; \mathrm{BMI} 27.6 \mathrm{~kg} \cdot \mathrm{m}^{-2}$ ) participated in the study. The subject was a member of the National Track Cycling Team specializing in sprint events. The participant was a medalist in international-level track races with a training experience of 11 years. The subject had not suffered from an injury or pain within the past six weeks.

\section{Experimental procedures}

The experiment was prepared during a class 1 international event (men's sprint), where the subject started in a $200 \mathrm{~m}$ flying start and four sprint races. Sprint races were divided into qualifying (one race), quarterfinal (one race), semifinals (two races) and finals (two races). During all races peak and averaged power output $\left(\mathrm{PO}_{\max }\right.$ and $\left.\mathrm{PO}_{\text {mean }}\right)[\mathrm{W}]$ and cadence $\left(\mathrm{C}_{\max }\right.$ and $\left.\mathrm{C}_{\text {mean }}\right)$ [rpm] were measured using PowerControl PC8 SRM (SRM GmbH, Germany). Rest HR was assessed using a Polar M400, heart rate monitor (Polar Electro, Finland). During the men's sprint events measurements of: a) peak and averaged heart rate $\left(\mathrm{HR}_{\max }\right.$ and $\left.\mathrm{HR}_{\text {mean }}\right)$ [bpm]; b) blood lactate (La-) [mmol. $\left.\right|^{-1}$ ] (Lactate Scout, SensLab GmbH, Germany); c) Borg rating of perceived exertion (RPE) - 20 point scale; d) viscoelastic properties of the muscles (muscle tone, stiffness, decrement, relaxation time and creep) (Myotonometer; MyotonPro, Myoton Ltd, Estonia); were taken at rest and in the $3^{\text {rd }}$ minute after each race. Time breaks between each stage were: 1) qualifying (200 m flying start) - quarterfinal (sprint 1) - $1^{\text {st }}$ semifinal (sprint 2): 
two hours; 2) $1^{\text {st }}$ semifinal race (sprint 2) $-2^{\text {nd }}$ semifinal race (sprint 3): 40 minutes; 3) $2^{\text {nd }}$ semifinal race (sprint 3) $-1^{\text {st }}$ final race (sprint 4): 1 hour.

A handheld Myotometer device was used to measure the lower extremity muscles viscoelastic properties (muscle tone, stiffness decrement, relaxation time and creep). Myotonometry measurements were made on the dominant lower extremity (right) at 23 reference points, including the anterior thigh muscles (points 1-7): rectus femoris (points 1-2); tensor fasciae latae (point 3); vastus lateralis (points 4-6); vastus medialis (point 7); posterior thigh muscles (points 8-15): external hamstrings (points 8-11); internal hamstrings (points 12-15); tibia muscles (points 16-18): tibialis anterior (point 16); peroneus (points 17-18); and posterior cuff muscles (points 19-23); external gastrocnemius (points 19-20); internal gastrocnemius (points 21-22); and soleus (point 23) muscles (Klich et al., 2018). For this study, three measurements at each reference point were performed, and the mean calculated. Measurement for one reference point took about $5 \mathrm{sec}$, while the total examination time was less than 2 min.

\section{Results}

The highest $\mathrm{PO}_{\max }$ and $\mathrm{PO}_{\text {mean }}$ were observed during the $200 \mathrm{~m}$ flying start and sprint 4 (1973 W; $1904 \mathrm{~W}$; $1172 \mathrm{~W}$ and $1124 \mathrm{~W}$, respectively). $C_{\text {max }}$ reached the highest value in sprint 4 (133 rpm), while $C_{\text {mean }}$ during the 200 $\mathrm{m}$ flying start and sprint 2 (103 rpm and $104 \mathrm{rpm}$ ). The subject reached the highest $\mathrm{HR}_{\max }$ noted during the $200 \mathrm{~m}$ flying start and sprint 2 (195 bpm both), and $\mathrm{HR}_{\text {mean }}$ in sprint 2 and 3 (184 bpm both). The highest La- was observed during the $200 \mathrm{~m}$ flying start and sprint $4\left(15.9 \mathrm{mmol} \cdot \mathrm{l}^{-1}\right.$ both). RPE increased during all starts, reaching the highest in sprint 4 (19) (Table 1).

Table 1. Power output, cadence and physiological parameters after men's sprints

\begin{tabular}{lcccccc}
\hline \multicolumn{1}{c}{ Variables } & Rest & $200 \mathrm{~m}$ & Sprint 1 & Sprint 2 & Sprint 3 & Sprint 4 \\
\hline $\mathrm{PO}_{\text {max }}[\mathrm{W}]$ & - & 1,973 & 1,632 & 1,778 & 1,668 & 1,904 \\
$\mathrm{PO}_{\text {mean }}[\mathrm{W}]$ & - & 1,172 & 1,034 & 1,045 & 1,103 & 1,124 \\
$\mathrm{C}_{\text {max }}[\mathrm{rpm}]$ & - & 129 & 124 & 126 & 129 & 133 \\
$\mathrm{C}_{\text {mean }}[\mathrm{rpm}]$ & - & 103 & 100 & 104 & 102 & 94 \\
$\mathrm{HR}_{\text {max }}[\mathrm{bpm}]$ & - & 195 & 186 & 195 & 191 & 189 \\
$\mathrm{HR}_{\text {mean }}[\mathrm{bpm}]$ & 70 & 182 & 178 & 184 & 184 & 177 \\
$\mathrm{La}\left[\mathrm{mmol} \cdot \mathrm{H}^{-1}\right]$ & 1.5 & 15.9 & 13.3 & 13.8 & 14.5 & 15.9 \\
$\mathrm{RPE}$ & 6 & 11 & 15 & 17 & 18 & 19 \\
\hline
\end{tabular}

Abbreviations: $\mathrm{PO}_{\max }=$ maximal power output $[\mathrm{W}] ; \mathrm{PO}_{\text {mean }}=$ mean power output $[\mathrm{W}] ; \mathrm{C}_{\max }=$ maximal cadency $[\mathrm{rpm}] ; \mathrm{C}_{\text {mean }}=$ mean cadency $[\mathrm{rpm}] ; \mathrm{HR}$ max $=$ maximal heart rate $[\mathrm{bpm}] ; \mathrm{HR}_{\text {mean }}=$ mean heart rate $[\mathrm{bpm}] ; \mathrm{La}^{-}=$lactate concentration $\left[\mathrm{mmol} \cdot \mathrm{I}^{-1}\right], \mathrm{RPE}=$ Rating of Perceived Exertion.

The average muscle tone and stiffness increased after the $200 \mathrm{~m}$ flying start and sprint 1 . The highest increase in tone observed in the hamstrings $(200 \mathrm{~m}-33.6 \%)$ and cuff (sprint $1-38.0 \%$ ), while stiffness in hamstrings (200 $\mathrm{m}-36.0 \%$ and sprint $1-47.7 \%$ ). In sprint 2 we observed a decrease in muscle tone (an average of 19.0\%) and stiffness (an average of 24.4\%). In the next sprints (sprint 3 and 4) we observed the highest increase in muscle tone and stiffness in the tibia (tone $-20.4 \%$ and $24.6 \%$; stiffness $-28.5 \%$ and $31.3 \%$ ), and decrease in stiffness (13.6\% and $15.0 \%)$. The highest decrement was observed in $1^{\text {st }}, 3^{\text {rd }}$ and $4^{\text {th }}$ sprints, especially in the anterior thigh ( $36 \% ; 39 \%$ and $52 \%$, respectively) and tibia muscles (33\%; $45 \%$ and $57 \%$, respectively) (Figure 1a-c). The average 
relaxation time and creep decreased after the $200 \mathrm{~m}$ flying start and sprint 1 . The highest decrease in relaxation time and creep observed in the hamstrings ( $R-31.6 \%$ and $44.2 \% ; C-31.3 \%$ and $45.0 \%$, respectively) and tibia ( $\mathrm{R}-31.1 \%$ and $43.0 \% ; \mathrm{C}-30.1 \%$ and $441 \%$, respectively). The relaxation time of the hamstrings increased (sprint $2-4)(12.4 \%$ and $19.4 \%$, respectively), however, decreased in the tibia (15.7\% and $20.9 \%$, respectively). The creep decreased in both muscle groups (Figure 2a, b).

a)
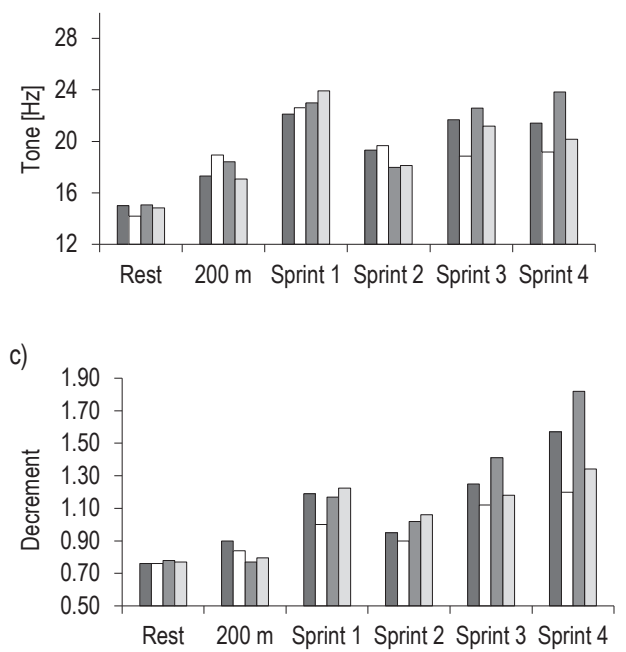

b)

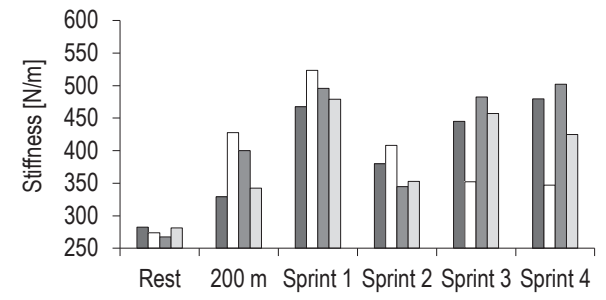

Figure 1. Muscle tone [Hz] (a), stiffness [N/m] (b) and decrement (c) after men's sprint
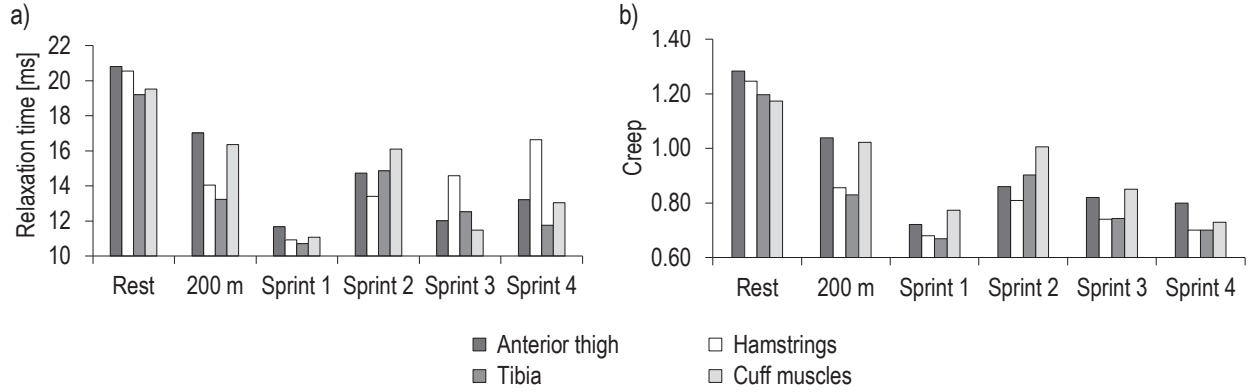

Figure 2. Relaxation time (a) and muscle creep (b) after men's sprint 


\section{Discussion}

Experimental measurements have demonstrated that the $200 \mathrm{~m}$ flying start race had a considerable impact as a substantial increase in muscle tone, stiffness, and a decrement in lower extremity muscles, especially in the hamstrings and tibia muscles. However, an increase in muscle tone, stiffness, and a decrement in the tibia muscles was observed after subsequent sprints. This event had the opposite result in muscle relaxation time and creep.

J. McDaniel et al. (McDaniel, Behjani, Elmer, Brown, Martin, 2014) have reported that during maximal isokinetic cycling at $120 \mathrm{rpm}, 49 \%$ of the power on the pedals was produced by the knee, $32 \%$ - by the hip, $9 \%$ - by the ankle, and transferred across the hip. Moreover, J.C. da Silva et al. (2016) observed that the quadriceps muscle and cuff muscle obtained the highest activity during cycling. Furthermore, the hamstrings can be activated during an increase in pedal rate. After the $200 \mathrm{~m}$ flying start and the $1^{\text {st }}$ sprint, there was an observed increase in muscle tone and stiffness, while after the $2^{\text {nd }}$ sprint a decrease in those parameters was found. The highest stiffness rate was observed in the hamstrings. Additionally, in sprints 3 and 4 a greater muscle stiffness was obtained, except for the hamstrings. R. Viir et al. (2007) reported a relationship between muscle tone and stiffness in the trapezius muscle. Those observations should be considered based on pedal stroke. Simultaneously an increase in anterior thigh stiffness is related to the propulsion phase, while during the downstroke phase the hamstrings and cuff muscles reach the highest stiffness (Dorel et al., 2005). The highest increase in tibia muscle stiffness, as well as a decrease in relaxation time and creep after the $3^{\text {rd }}$ and the $4^{\text {th }}$ sprint was caused probably by a high range of action (all the range) and peak activity angle $\left(280^{\circ}\right)$ (Ryan, Gregor, 1992). A. White et al. (2018) found a correlation between relaxation time and creep of lower lumbar erector spinae muscle. From the biomechanical point of view, as muscle stiffness increases, relaxation time shortens, and less degree of creep is needed. Furthermore, stiffness is inversely proportional to relaxation time, because the stiffer the tissue, the shorter time is needed to recover. According to C.R. Abbiss et al. (Abbiss, Peiffer, Laursen, 2009), a sprint cyclist's pedal rate should be about 100-120 rpm, as it reduces neuromuscular fatigue. In our opinion, sprint tactics and increased muscle fatigue could influence the alterations in viscoelastic properties. Increase in pedaling rate could be associated with greater activity of the vastus medialis, medial hamstrings and the calf muscles. The last sprint ( $\left.4^{\text {th }}\right)$ was followed by a maximal cadence of $133 \mathrm{rpm}$, while the mean cadence was $94 \mathrm{rpm}$. T. Takaishi et al. (Takaishi, Yasuda, Ono, Moritani, 1996) suggest that lower pedaling rate may lead to worse blood flow and venous return in the muscles. An increase in decrement could confirm our observations, because this parameter reflects muscle elasticity. Biomechanically, decrement is inverse proportional to elasticity. A. White et al. (2018) have reported a positive correlation between muscle stiffness and decrement. In our opinion, alterations in viscoelastic properties in the tibia muscles are related to increased fatigue of the anterior thigh and cuff muscles (Klich et al., 2018).

\section{Conclusion}

The $200 \mathrm{~m}$ flying start and repeated sprint races appear to affect the viscoelastic properties of lower extremity muscles in track cyclists. The increase of muscle tone, stiffness and decrement is followed by a decrease in relaxation time and creep. This study has proved that the muscle fatigue mechanism is related to alterations in viscoelastic properties. Moreover, fatigue mechanisms might influence muscle outcomes, e.g. muscle activation, recruitment and fibers type. This research presents the individual results of a single athlete, yet the main findings of the study could be useful information in programming specific workouts and recovery sessions. 


\section{Referencences}

Abbiss, C.R., Peiffer, J.J., Laursen, P.B. (2009). Optimal cadence selection during cycling. International SportMed Journal, 10 (1), 1-15.

da Silva, J.C., Tarassova, O., Ekblom, M.M., Andersson, E., Ronquist, G., Arndt, A. (2016). Quadriceps and hamstring muscle activity during cycling as measured with intramuscular electromyography. Eur J Appl Physiol, 116 (9), 1807-1817.

Davidson, M.J., Bryant, A.L., Bower, W.F., Frawley, H.C. (2017). Myotonometry Reliably Measures Muscle Stiffness in the Thenar and Perineal Muscles. Physiother Can, 69 (2), 104-112.

Dorel, S., Hautier, C.A., Rambaud, O., Rouffet, D., Van Praagh, E., Lacour, J.R., Bourdin, M. (2005). Torque and power-velocity relationships in cycling: relevance to track sprint performance in world-class cyclists. Int J Sports Med, 26 (9), 739-746.

Kawczyński, A., Mroczek, D., Andersen, R.E., Stefaniak, T., Arendt-Nielsen, L., Madeleine, P. (2018). Trapezius viscoelastic properties are heterogeneously affected by eccentric exercise. J Sci Med Sport, 21 (8), 864-869.

Kawczyński, A., Samani, A., Mroczek, D., Chmura, P., Blach, W., Migasiewicz, J., Klich, S., Chmura, J., Madeleine, P. (2015). Functional connectivity between core and shoulder muscles increases during isometric endurance contractions in judo competitors. Eur J Appl Physiol, 115 (6), 1351-1358.

Klich, S., Krymski, I., Michalik, K., Kawczyński, A. (2018). Effect of short-term cold-water immersion on muscle pain sensitivity in elite track cyclists. Phys Ther Sport, 32, 42-47.

Martin, J.C., Davidson, C.J., Pardyjak, E.R. (2007). Understanding sprint-cycling performance: the integration of muscle power, resistance, and modeling. Int J Sports Physiol Perform, 2 (1), 5-21.

McDaniel, J., Behjani, N.S., Elmer, S.J., Brown, N.A., Martin, J.C. (2014). Joint-specific power-pedaling rate relationships during maximal cycling. J Appl Biomech, 30 (3), 423-430.

Ryan, M.M., Gregor, R.J. (1992). EMG profiles of lower extremity muscles during cycling at constant workload and cadence. J Electromyogr Kinesiol, 2 (2), 69-80.

Stafilidis, S., Arampatzis, A. (2007). Muscle - tendon unit mechanical and morphological properties and sprint performance. J Sports Sci, 25 (9), 1035-1046.

Takaishi, T., Yasuda, Y., Ono, T., Moritani, T. (1996). Optimal pedaling rate estimated from neuromuscular fatigue for cyclists. Med Sci Sports Exerc, 28 (12), 1492-1497.

Viir, R., Virkus, A., Laiho, K., Rajaleid, K., Selart, A., Mikkelsson, M. (2007). Trapezius muscle tone and viscoelastic properties in sitting and supine positions. Scandinavian Journal of Work, Environment \& Health, 33 (3), 76.

White, A., Abbott, H., Masi, A.T., Henderson, J., Nair, K. (2018). Biomechanical properties of low back myofascial tissue in younger adult ankylosing spondylitis patients and matched healthy control subjects. Clin Biomech (Bristol, Avon), 57, 67-73.

Cite this article aS: Klich, S., Krymski, I., Kawczyński, A. (2019). Viscoelastic Properties of Lower Extremity Muscles after Elite Track Cycling Sprint Events: A Case Report. Central European Journal of Sport Sciences and Medicine, 1 (29), 5-10. DOI: 10.18276/cej.2020.1-01. 